\title{
Current and Emerging Treatment Options for Castration-Resistant Prostate Cancer: A Focus on Immunotherapy
}

\author{
Winald R. Gerritsen • Padmanee Sharma
}

Received: 15 August 2011 / Accepted: 12 September 2011 /Published online: 4 November 2011

(C) The Author(s) 2011. This article is published with open access at Springerlink.com

\begin{abstract}
Background Castration-resistant prostate cancer is a disease with limited treatment options. However, the ongoing elucidation of the mechanisms underlying this disease continues to support the development of not only novel agents, but also innovative approaches. Among these therapies, immunotherapy has emerged as a promising strategy.

Design This review article summarizes the most recent data from investigations of immunotherapies in castrationresistant prostate cancer (literature and congress searches current as of August 2011).

Results Immunotherapeutic strategies such as passive immunization, vaccines, and particularly checkpoint blockade have demonstrated some efficacy as single agents. Elucidation of effective combinations of agents and drug regimens is ongoing but will require continued careful investigation, including the standardization of surrogate endpoints in clinical trials.

Conclusions It is hypothesized that the combination of immunotherapeutic agents with traditional and novel chemotherapeutics will potentiate the efficacy of the chemotherapeutics while maintaining manageable toxicity.
\end{abstract}

Keywords Biochemotherapy · castration-resistant prostate cancer - chemotherapy $\cdot$ hormone-refractory prostate cancer . immunotherapy

W. R. Gerritsen $(\square)$

Department of Medical Oncology, VU University Medical Center, PO Box 7057, 1007MB, Amsterdam, Netherlands

e-mail: winald.gerritsen@vumc.nl

\section{P. Sharma}

Department of Genitourinary Medical Oncology,

Division of Cancer Medicine,

The University of Texas, M. D. Anderson Cancer Center,

Houston, USA

\section{Introduction}

Prostate cancer is the most common nonskin cancer in males in the USA. In 2009, an estimated 192,280 cases were diagnosed, with 27,360 deaths attributed to the disease [1]. Certain low-risk cases of clinically localized prostate cancer can be managed by active surveillance, but most patients are initially managed with radiotherapy (external beam and interstitial), radical prostatectomy, or cryosurgery [2]. Advanced prostate cancer requires androgen deprivation therapy (ADT) as the standard of care. However, although the majority of men initially respond to ADT, most tumors become resistant to primary hormone therapy, with a median time to androgen independence of 14 to 30 months [3]. Castration-resistant prostate cancer (CRPC) is defined as progressive disease in a patient with a castrate level of testosterone [4]. For men with CRPC, chemotherapy with docetaxel has been proven to extend survival and is considered standard of care for patients [5, 6]. In 2010, the autologous cellular immunotherapeutic sipuleucel-T (PROVENGE $^{\circledR}$, Dendreon Corporation, Seattle, WA, USA) also received Food and Drug Administration (FDA) approval in patients with asymptomatic or minimally symptomatic metastatic CRPC based on a survival advantage in phase III testing, and this approval provides firm proof of principal for immunotherapy in CRPC. Cabazitaxel (JEVTANA ${ }^{\circledR}$, Sanofi-aventis, Bridgewater, NJ, USA), a microtubule inhibitor, was also approved by the US FDA in 2010 to be used in combination with prednisone for treatment of patients with CRPC previously treated with a docetaxel-containing treatment regimen. In addition to these interventions, significant clinical research has been directed at identifying novel therapies that prolong survival while maintaining quality of life of patients with CRPC, and it is predicted that this body of research will continue to 
augment the treatment options available to these men. This review will provide a background of the strategies that are currently under development for CRPC (including chemotherapy, radiotherapy, and immunotherapy), followed by a discussion about immunotherapy-focused combination approaches and important considerations for clinical trial development of these strategies.

\section{Nonimmunotherapeutic Options for CRPC}

The goal of current treatments for CRPC is to stop or slow the growth of cancer cells in order to prolong patient survival without negatively affecting their quality of life. Mitoxantrone was the first chemotherapeutic agent that demonstrated clinical activity in CRPC, approved in 1996 based on an improvement in quality of life [7]. Two landmark trials, SWOG9916 and TAX327, which compared mitoxantrone against docetaxel, established docetaxel as the current standard of care for patients for whom chemotherapy is indicated. These trials showed improvements in time-to-progression (TTP), prostate-specific antigen (PSA) response, a lowered hazard ratio (HR) for death, and an improved survival benefit in the docetaxel arms [5, 6], which led to US FDA approval for docetaxel-based therapy for CRPC.

Cabazitaxel was studied in the phase III TROPIC study $(N=755)$, which compared the safety and efficacy of cabazitaxel plus prednisone $(\mathrm{CP})$ to mitoxantrone plus prednisone (MP) in the treatment of CRPC, which had progressed after docetaxel use. Treatment with $\mathrm{CP}$ reduced the risk of death by $30 \%(\mathrm{HR}=0.70 ; p<0.0001)$ [8]. There was a statistically significant benefit in median overall survival (OS) in the CP group (15.1 months for the CP group compared with 12.7 months in the MP group) [8]. A phase III study is presently underway to compare OS with differing doses of CP in metastatic CRPC patients, postdocetaxel chemotherapy (NCT01308580). Additionally, a second phase III study is evaluating OS in chemotherapynaive metastatic CRPC patients treated with two different doses of CP regimen, in comparison with a third arm where patients will receive docetaxel and prednisone therapy (NCT01308567).

An increased understanding of the molecular mechanisms and pathways that control the development of CRPC has led to the development of new therapeutic agents, some general cytotoxic agents and others that are directed at key targets of these pathways. Therapy with agents that specifically target proteins that drive CRPC may have fewer side effects than those caused by cytotoxic agents like docetaxel. A myriad of treatment options are in development but none have yet shown significant advantages over docetaxel.
Therapies that target the androgen receptor (AR) axis more efficiently (e.g., abiraterone, MDV3100) are promising agents in development for CRPC. Abiraterone acetate (AA) is a potent steroidal inhibitor of CYP17 (17 alphahydroxylase/C17,20-lyase), blocking two important enzymatic activities in the synthesis of testosterone. AA (ZYTIGA $^{\mathrm{TM}}$, Centocor Ortho Biotech Inc, Horsham, PA, USA) was approved by the US FDA and is expected to be approved by the European Medicines Agency also in 2011 for use in combination with prednisone in the treatment of metastatic CRPC in men who have received prior docetaxel chemotherapy. Recently, a pivotal randomized phase III trial, comparing $\mathrm{AA}$ and prednisone versus prednisone alone in CRPC patients who have progressed on docetaxel, showed improved OS in the AA arm with a median survival of 14.8 months compared with 10.9 months in the placebo arm [9]. Improvements in radiographic progression-free survival (rPFS), time to PSA progression, and PSA response rates were also observed. A phase III study comparing AA plus prednisone versus placebo plus prednisone is ongoing in men with CRPC who are asymptomatic or minimally symptomatic and chemotherapy-naive (NCT00887198).

MDV3100 is a small molecule AR antagonist that blocks nuclear translocation of AR DNA binding [10]. Following promising results from a phase I/II trial with MDV3100, two large randomized, multicenter phase III trials are currently underway, comparing MDV3100 versus placebo in chemotherapy-refractory or -naive CRPC patients (NCT00974311 and NCT01212991, respectively). An interim analysis of the latter trial in 65 chemotherapynaive CRPC patients showed that the circulating tumor cell (CTC) number, PSA level, and PSA response at 12 weeks were significant predictors of rPFS [11].

TAK-700 is a nonsteroidal androgen synthesis inhibitor of 17,20-lyase, which is a key enzyme in the production of steroidal hormones. Currently, two phase III studies in CRPC patients are underway, comparing the combination of TAK-700 plus prednisone versus placebo and predisone in both chemotherapy-naive (NCT01193244) and postchemotherapy (NCT01193257) settings. Additionally, TOK-001, a dual antiandrogen and CYP17 inhibitor, is in clinical development [12].

Other strategies for disrupting the AR signaling pathway are in early stages of development for the treatment of CRPC. These include inhibition of heat shock proteins, such as HSP90 (required for the folding and stabilization of the AR), and the use of histone deacetylase inhibitors to suppress the transcription of AR and AR-related genes. These agents have yet to show much antitumor activity but further investigation in combination with other agents may prove their clinical benefit.

Epothilones (e.g., ixabepilone, patupilone) inhibit cell division by interfering with microtubule function and have 
shown promise in the treatment of CRPC. In a small phase II trial of chemotherapy-naive CRPC patients receiving ixabepilone $\left(40 \mathrm{mg} / \mathrm{m}^{2}\right)$ every 3 weeks, $14(33 \%)$ achieved a PSA decline [13]. In the responders, $72 \%$ had a $>80 \%$ decline in PSA, and 2 patients achieved an undetectable PSA. Ixabepilone alone has demonstrated modest antitumor activity in CRPC, and ongoing studies of this agent in combination with other therapies may demonstrate activity that exceeds that of ixabepilone monotherapy.

\section{Prostate Cancer: An Attractive Target for Immunotherapy}

Unlike a chemotherapeutic agent that can take an immediate effect on its target, immunotherapy requires time for the patient's immune system to mount a response. Thus, immunotherapy is well suited for a slow-growing disease such as prostate cancer [14], in which it is reasonable to allow time for a clinically meaningful immune response to occur. Multiple proteins unique to the prostate can serve as antigens, and immunotherapies that exploit these antigens may be able to direct an immunologic response specifically to a prostate tumor. Equally important is the fact that prostate glands are infiltrated by CD4 and CD8 T-cell populations that are oligoclonally expanded (which suggests their presence is not random, but rather the result of a specific antigenic stimulation) $[15,16]$. Abundant preclinical studies have shown that immunological responses in the prostate are elicited by various therapies, including immune checkpoint blockade and radiotherapy. Interestingly, therapies to which prostate cancer is initially responsive, such as hormonal manipulation and androgen withdrawal, have been shown to induce T-cell infiltration in prostate glands and tumors [15].

Currently, most studies are investigating the efficacy of immunotherapy in patients with metastatic CRPC, a disease in which many tumor-induced immunosuppressive mechanisms have already been established. Biochemical recurrence, as evidenced by rising PSA levels after surgery or androgen ablation, provides a unique opportunity for immunological intervention before the immunosuppressive mechanisms associated with advanced disease are in place. Therefore, early intervention with immunotherapy and other agents may prove to be more effective than currently approved and tested strategies.

\section{Emerging Immunotherapeutic Approaches for CRPC}

Immunotherapy for CRPC is an active field of investigation. A large number of immunotherapy approaches are being developed in prostate cancer with the goal of inducing a specific T-cell response directed against the tumor. Multiple tumor-associated antigens (e.g., PSA, prostate-specific membrane antigen [PSMA], and prostatic acid phosphatase [PAP]) have been identified as potential targets for prostate immunotherapy. The two major approaches of immunotherapy are passive (agent has intrinsic immunologic activity) and active (agent is designed to stimulate host's immune response).

One example of passive immunotherapy is using radiolabeled monoclonal antibodies against tumor-specific proteins to target radiation directly to the tumor. Radiolabeled J591 (monoclonal antibody directed against PSMA) using the radionuclides yttrium- 90 and lutetium-177 has demonstrated manageable myelotoxicity, no significant nonhematologic toxicity, excellent tumor targeting (both of soft tissue and bone metastases), and preliminary evidence of efficacy, including PSA declines and measurable disease responses $[17,18]$. A dose escalation phase II trial of 177Lu-J591 in 30 patients with metastatic CRPC reported 3 patients with $\geq 50 \%$ decline in PSA and 9 with $\geq 30 \%$ decline with more responders at higher dose. As a result of this therapy, nine patients required one to four platelet transfusions [19]. The preliminary results in this small clinical trial are being confirmed in ongoing studies of J591 as a monotherapy and in combination with docetaxel (NCT00195039 and NCT00916123, respectively).

Vaccine-based therapies seek to directly stimulate a specific immune reaction against a single or many tumor antigens. While the methods vary, vaccine therapies aim to drive a specific antitumor response without compromising normal tissues [3]. The four main types of vaccines that have been investigated for CRPC can be classified as viral vector based, cell based, DNA vaccines, and autologous (derived from a patient's own tumor cells).

Viral vaccination is an approach in which a gene (e.g., that encoding PSA) is inserted into a recombinant virus vector, after which a patient is immunized with a live, attenuated recombinant virus. A recombinant vaccinia virus, which was widely applied for vaccination against smallpox, was modified to express PSA (rV-PSA; PROSTVACTM, Bavarian Nordic A/S, Kvistgård, Denmark). The vaccine was well tolerated and induced durable PSA level decreases in nearly half of the patients tested [20, 21]. However, repeated doses of rV-PSA were proven to be ineffective at inducing PSAspecific T-cell populations, possibly due to an immune response against the vaccinia virus [21]. Consequently, a prime/boost vaccine strategy (PROSTVAC-VF) was tested using vaccinia virus and fowlpox virus (which will infect but will not replicate in mammalian cells and may allow for prolonged gene expression as compared with vaccinia) expressing human PSA. In this study, 64 patients were randomized to 3 treatment cohorts; $45.3 \%$ of patients remained free of PSA progression at 19.1 months and 
$78.1 \%$ demonstrated clinical progression-free survival (PFS) [22].

The PROSTVAC-VF vaccine was advanced by the addition of three virally expressed T-cell costimulatory molecules: B7-1, intercellular adhesion molecule-1, and lymphocyte function-associated antigen-3 (TRICOM) [23]. A phase II study of 122 patients with CRPC was carried out, where patients were randomized to placebo or PROSTVAC-VF/TRICOM+granulocyte-macrophage colony-stimulating factor (GM-CSF) treatments. GM-CSF, a cytokine adjuvant, is frequently administered with vaccines, since it promotes maturation and expression of major histocompatibility complexes (MHCs) and costimulatory molecules by antigen-presenting cells (APCs), in addition to recruiting granulocytes and augmenting T-cell response [24]. In the trial, PFS was similar in the treatment and placebo groups $(p=0.6)$ [25]. However, at 3 years poststudy, 25 (30\%) vaccine patients were alive compared with $7(17 \%)$ control patients. Patients in the vaccine arm had a longer median survival than those in the control arm (25.1 and 16.6 months, respectively; estimated $\mathrm{HR}=0.56$; $p=$ 0.0061 ). These results need to be confirmed in a larger phase III study.

GVAX is a cell-based allogeneic vaccine consisting of the $\mathrm{LNCaP}$ and $\mathrm{PC}-3$ prostate cell lines genetically engineered to secrete high levels of GM-CSF. These cells are injected intradermally in order to initiate an antiprostate immune response. A phase II dose escalation study in 80 men with CRPC showed that PSA stabilization occurred in $15(19 \%)$ patients, and a $>50 \%$ decline in PSA was seen in 1 patient [26]. Median survival times were 35.0 months in the high-dose group, 20.0 months in the mid-dose group, and 23.1 months in the low-dose group. The proportion of patients who generated an antibody response to one or both cell lines increased with dose and included 10 of 23 (43\%) in the low-dose group, 13 of $18(72 \%)$ in the mid-dose group, and 16 of $18(89 \%)$ in the high-dose group ( $p=$ 0.002). Based on these observations, the highest dose was used for further clinical studies. Two phase III trials were designed to study GVAX in asymptomatic and symptomatic men with metastatic CRPC. The Vaccine Immunotherapy with Allogeneic Prostate Cancer Cell Lines (VITAL-1) trial compared GVAX to prednisone plus docetaxel in men with asymptomatic CRPC. This trial finished accrual of patients and was terminated by Cell Genesys, Inc. because a futility analysis indicated that there was $<30 \%$ chance of achieving a survival benefit. VITAL-2 compared the combination of GVAX and docetaxel treatment with the use of prednisone and docetaxel in symptomatic CRPC. VITAL-2 was terminated in August 2008 due to an imbalance in deaths in the two arms, with 67 deaths in the GVAX and docetaxel arm compared with 47 in the control group [27].
Other studies have examined DNA vaccines with PSA to induce immune responses. DNA vaccines have the advantage of ease of production and administration, as well as lack of viral antigens that may generate an immune response. However, a disadvantage with DNA vaccines includes the inability of producing a strong immune response due to low cellular transfection efficiency [3]. A DNA vaccine encoding PAP is in the early stages of development for the treatment of CRPC. A phase I/IIa dose escalation trial of this vaccine coadministered intradermally with the adjuvant GM-CSF was conducted in patients with stage D0 prostate cancer (biochemical [PSA] recurrence after definitive local therapy) [28]. Patients developed PAPspecific T-cell immune response shortly after the treatment course, as determined by enzyme-linked immunospot. The median PSA doubling time was observed to increase from 6.5 months pretreatment to 8.5 months on-treatment $(p=$ $0.033)$ and 9.3 months in the 1-year post-treatment period $(p=0.054)$. A minimal adverse event (AE) profile was observed and a larger trial of clinical efficacy is warranted. Additionally, the University of Wisconsin is currently sponsoring a phase II pilot study to determine the safety of serial intradermal vaccinations PAP with GM-CSF as an adjuvant, as well as to determine if the vaccine generates long-lived immune responses in patients with nonmetastatic CRPC (NCT00849121).

Dendritic cells, which are widely distributed in nearly all tissues, present antigens through their MHC class 1 and 2 receptors and thus can induce immune responses by activating $\mathrm{T}$ cells to develop a potent antitumor response. Autologous dendritic cells can be grown in vitro and transfected with antigen, cytokines, or other agents before reintroduction to the patient to direct an immune response [3]. Sipuleucel-T (APC8015) is a widely used dendritic cell vaccine comprised of peripheral blood mononuclear cells (including APCs) and pulsed ex vivo with a proprietary PAP-GM-CSF fusion protein. A phase III trial of 127 men with CRPC randomized to APC8015 or placebo was conducted [29]. Although treatment with sipuleucel-T did not result in a statistically significant delay in TTP, there was a statistically significant $(p=0.01)$ survival advantage of 4.5 months in an intent-to-treat analysis. A recent phase III trial, Immunotherapy for Prostate Adenocarcinoma Treatment compared sipuleucel- $T$ with placebo in 512 men with CRPC [30]. The sipuleucel-T-treated group demonstrated $22 \%$ reduction in the risk of death as compared with the placebo group (HR $=0.78 ; 95 \%$ confidence interval, 0.61-0.098; $p=0.03$ ), which represented a 4.1-month (25.8 versus 21.7 months) increase in OS, the primary study endpoint. The estimated 36-month rates of survival were $32 \%$ and $23 \%$ for the sipuleucel-T and placebo groups, respectively. AEs that were more frequently reported in the sipuleucel-T group than in the placebo 
group included chills (51\%), fever (31.3\%), and headache $(18.1 \%)$; these were grade 2 or less in all but $3.5 \%$ of patients. Based on these results, this vaccine was approved by the FDA for the treatment of men with asymptomatic or minimally symptomatic CRPC.

Another type of immunotherapy utilizes immunomodulators to affect immune responses. The goal of immunomodulation in CRPC is to stimulate the immune system to attack prostate cancer cells. One such immunomodulator is GM-CSF, a pluripotent cytokine that has manifold impact on the immune system, including enhancing T-cell responses. GM-CSF first showed efficacy in CRPC by decreases in PSA levels leading to long-term disease control in $\sim 15 \%$ of treated patients $[31,32]$. A study of GM-CSF in combination with ipilimumab is discussed in the "Combination Strategies" section.

Monoclonal antibodies that target proteins important in modulating immune response represent another promising type of immunotherapy for CRPC. Antibodies can be used to block negative immunological checkpoints that impede an effective immune response (such as cytotoxic Tlymphocyte antigen-4 [CTLA-4] and programmed death-1 [PD-1]) or enhance stimulatory signals (like 41BB). The goal of this "checkpoint blockade" therapy is to sustain and boost an antitumor immune response by enabling the patient's immune system to overcome the innate regulatory mechanisms that control immune responses.

Ipilimumab (YERVOYTM, Bristol-Myers Squibb, Princeton, NJ, USA) is an antibody directed against CTLA-4, a key negative regulator of $\mathrm{T}$ cells [33] that was approved in March 2011 by the US FDA for metastatic melanoma treatment. In a presurgical clinical trial, the antibody has been shown to lead to infiltration of effector $\mathrm{T}$ cells into tumor tissues with concomitant decreases in regulatory $\mathrm{T}$ cells, thus increasing the ratio of effector to regulatory $\mathrm{T}$ cells within the tumor microenvironment $[34,35]$. In a pilot study involving 14 patients with metastatic CRPC, 2 patients had $\geq 50 \%$ decline in PSA. The most common AEs observed with ipilimumab use are related to its immune-based mechanism of action; these AEs were usually inflammatory in nature and different from AEs observed with chemotherapies or other immunotherapies. The treatment was well tolerated in all but one patient who developed a grade $3 \mathrm{rash} /$ pruritus [36]. Given promising results of ipilimumab use in metastatic CRPC patients from this and other phase I/II studies [36-39], a randomized, double-blind, phase III trial to compare the efficacy of ipilimumab versus placebo in patients with metastatic chemotherapy-naive CRPC is currently underway, where comparison of OS between the two groups is a primary endpoint (NCT01057810).

PD-1 and its ligands PDL-1 and PDL-2 represent another immune checkpoint pathway of noted clinical interest. Early clinical testing has demonstrated that PD-1 blockade has considerable activity against a variety of advanced solid tumors, including renal carcinomas [24, 39]. Two phase I trials examining the safety of a fully human monoclonal anti-PD-1 antibody, MDX-1106 (NCT00441337 and NCT00730639), include advanced prostate cancer patients, though no clinical responses have yet been reported in prostate cancer patients in these trials [40, 41].

\section{Combination Strategies}

Although immunotherapy has shown promising responses as monotherapy, we and others believe that combination therapy with standard modalities and novel nonimmunotherapeutic, as well as immunotherapeutic agents, will be necessary to provide improved antitumor responses and clinical benefit. Multiple ongoing trials are testing various combinations of the strategies discussed above. In the immunotherapy arena, strategies that are the focus of multiple clinical trials include combination of vaccines and chemotherapy, vaccines and androgen ablation, immunotherapy and radiotherapy, and checkpoint blockade agents (e.g., ipilimumab) with various treatments.

The combination of vaccine with chemotherapy has demonstrated clinical benefit, albeit it in a small study. CRPC patients $(N=28)$ were randomized to receive either vaccine (rV-PSA admixed with rV-B7.1 as a prime, followed by rF-PSA booster) and weekly docetaxel or vaccine alone [42]. Eleven patients who progressed on vaccine alone crossed over to receive docetaxel at the time of progression. Receiving the vaccine prior to treatment with docetaxel enhanced survival, over that observed historically with docetaxel treatment. An interesting subanalysis of two sipuleucel-T trials suggested that this vaccine enhances the effect of docetaxel [43]. Among patients who received sipuleucel-T followed by docetaxel, the median survival was 34.5 months; patients who were on placebo then docetaxel had a survival of 25.7 months and patients treated with docetaxel alone had a median survival of 20.2 months. Larger phase II trials to test combinations of different vaccines and chemotherapeutic agents are ongoing (NCT01194960 and NCT01145508).

Androgen ablation can have a stimulatory effect on the immune response, including the infiltration of activated $\mathrm{CD} 4+\mathrm{T}$ cells into the prostate gland and the induction of antigen-specific immune responses [15, 44]. Androgen ablation is presently being evaluated in combination with ipilimumab, compared with androgen ablation alone, in advanced prostate cancer patients in a phase II trial with the rationale that the two agents may act synergistically in that androgen ablation induces an intense T-cell infiltrate in the prostate and CTLA-4 blockade potentiates T-cell responses 
[45]. Initial results show that the concurrent administration of ipilimumab with androgen ablation may be synergistic as hypothesized, with patients in the combination therapy group more likely to have undetectable PSA by 3 months compared with the androgen ablation only group $(55 \%$ versus 38\%) [45]. The timing of androgen ablation in combination with other therapies may prove to be crucial. One study demonstrated that responses to a combination of vaccine therapy, GM-CSF, and interleukin-2, were better in patients that received androgen ablation after receiving the vaccine than in those who received it before vaccine therapy [46]. The immunologic effect of radiotherapy in patients with prostate cancer has recently been shown [47]. The mechanism is thought to involve the priming of an antitumor immune response as irradiation induces a proinflammatory response in which dying tumor cells expose tumor antigens to infiltrating immune cells [48, 49]. A randomized phase II trial tested the combination of a poxviral vaccine encoding PSA and radiotherapy in patients with clinically localized prostate cancer. Thirteen out of 17 patients in the combination arm had increases in PSAspecific $\mathrm{T}$ cells of at least threefold versus no detectable increases in the radiotherapy-only arm $(p<0.0005)$ [50]. The combination of radiotherapy and the immunomodulator ipilimumab is discussed below.

The checkpoint blockade agent ipilimumab is being studied in combination with immunomodulators (GMCSF), vaccines (GVAX), radiotherapy, and chemotherapy. GM-CSF in combination with ipilimumab was tested in 24 patients with CRPC [37]. In this study, three of six patients treated at the highest level of ipilimumab had $>50 \%$ PSA declines; one of these patients showed partial response in visceral metastases. This combination was shown to have an immunostimulatory effect as it induced the expansion of activated effector CD8 $\mathrm{T}$ cells in vivo. These results need to be confirmed and the impact on survival should be determined in a larger trial.

A phase I trial of GVAX in combination with ipilimumab is underway and preliminary results show the therapy is well tolerated and active in CRPC [51]. Twelve patients were treated in a dose escalation phase for 24 weeks with biweekly intradermal injections of GVAX and monthly ipilimumab at $0.3,1,3$, or $5 \mathrm{mg} / \mathrm{kg}$. Late onset PSA responses (declines $>50 \%$ ) were seen in these five patients with response durations of 6.7, 8.6, 9.5, 13.8 (ongoing), and 23.1 months. Four of these patients had stable disease on bone scan for at least 12 months and up to 21 months. Five of six patients at the higher ipilimumab doses ( 3 and $5 \mathrm{mg} /$ $\mathrm{kg}$ ) developed grade 2 or 3 ipilimumab-related AEs. Follow-up on the 16 expansion cohort patients, as well as a larger clinical trial with an optimal dose of ipilimumab, will provide more robust data on safety and clinical activity in prostate cancer.
In a phase I dose escalation trial of ipilimumab $(3,5$, and $10 \mathrm{mg} / \mathrm{kg}$ ) alone and in combination with single fraction radiotherapy in patients with metastatic CRPC, 6 of 26 treated patients (23\%) had a confirmed $>50 \%$ PSA decline. One of the seven patients with measurable disease had a partial response in nodal metastases and achieved an undetectable PSA after treatment with $10 \mathrm{mg} / \mathrm{kg}$ [38]. A phase II study of $10 \mathrm{mg} / \mathrm{kg}$ ipilimumab alone or with radiation in 45 patients with CRPC reported 10 patients (22\%) with confirmed PSA declines $\geq 50 \%$ [39]. The median time to PSA decline was 5.7 weeks and the median duration of PSA decline was 23 weeks. One patient dosed with ipilimumab alone experienced a PSA level $\leq 0.05 \mathrm{ng} /$ $\mathrm{ml}$, as well as complete responses of bone, nodal, and prostate lesions which continued for $54+$ and $84+$ weeks, respectively. Ipilimumab-associated AEs were experienced in 17 patients (38\%) and included diarrhea/colitis, rash/ pruritus, hepatitis, and endocrinopathy. Eleven patients (24\%) experienced $\geq$ grade 3 events (gastrointestinal [9 patients; 20\%] and hepatitis [4 patients; 9\%]), and all resolved with corticosteroid and/or infliximab therapy. A randomized phase III trial comparing combination use of radiotherapy and ipilimumab to radiotherapy and placebo in men who have failed docetaxel chemotherapy is currently underway (NCT00861614).

In a small trial testing the combination of ipilimumab and a single dose of docetaxel in patients with CRPC, there was no apparent enhancement of activity by coadministration of a single dose of docetaxel [52]. However, the combination of ipilimumab and the chemotherapeutic agent dacarbazine demonstrated more clinical activity than ipilimumab in melanoma patients [53], suggesting that the combination of ipilimumab and chemotherapy may be clinically beneficial and should be further explored in prostate cancer.

\section{Challenges to the Development of Novel Therapies for CRPC}

The failures of the clinical trial programs for many drugs in development for CRPC demonstrate the difficulties in finding an effective treatment for this disease. For example, while the oral platinum analog satraplatin initially showed an improved in PFS of 2.5 to 5.2 months [54], it failed to demonstrate an OS benefit in a large phase III trial [55]. The endothelin A receptor antagonist atrasentan failed to show improvement in TTP and median survival times when compared with placebo [56]. Furthermore, combinations of docetaxel with other agents have showed either limited efficacy or unacceptable toxicity. A recent small study of docetaxel and $10 \mathrm{mg}$ atrasentan reported OS and PFS comparable to docetaxel and prednisone [57]. The phase II 
Androgen-Independent Prostate Cancer Study of Calcitriol Enhancing Taxotere-1 (ASCENT-1) study, which investigated the use of docetaxel in combination with the vitamin D agonist DN-101, failed to achieve its primary endpoint of improved PSA response rate; however, this study initially appeared to demonstrate an increased median survival benefit with combination therapy [58]. A larger ASCENT2 study, which began enrolling patients in February 2006, was halted early and eventually terminated due to the excessive number of deaths in the treatment arm compared with the control arm [58]. Lastly, the study of the combination of docetaxel and the vaccine GVAX was closed early because of excess deaths in the GVAX combination arm (as compared with GVAX alone) [27, 58].

The development of an effective therapy for men with CRPC has been challenged by both the nature of the disease and by the lack of reliable assessment endpoints to determine the efficacy of novel agents. For example, less than half of men with CRPC have measurable target lesions $>2 \mathrm{~cm}$ in size. Therefore, it has been recommended that trials should no longer require that a patient has measurable lesions (target lesions as defined by Response Evaluation Criteria in Solid Tumors [RECIST]) for entry [4]. It is also difficult to determine a favorable outcome in bone, the most common site of prostate cancer spread. Consequently, there is a lack of a reliable and identifiable surrogate endpoint for OS in prostate cancer clinical trials.

The most widely accepted efficacy marker in CRPC clinical trials is PSA. The PSA level is related to tumor growth and generally has a positive correlation with tumor burden; however, a correlation between a given posttherapy change and true clinical benefit has not been fully defined. While PSA level itself is not a reliable endpoint, PSA kinetics (slope, PSA doubling time) may prove to be. In the search for reliable surrogate endpoints for clinical trials in prostate cancer, researchers have also evaluated the following endpoints: CTC count, alkaline phosphatase and lactate dehydrogenase levels, bone scans, symptoms assessment, and computed/tomography/magnetic resonance imaging scans.

The second Prostate Cancer Clinical Trials Working Group (PCWG2) recently reassessed the outcome measures for clinical trials in prostate cancer. The 2008 guidelines recommend that outcomes are reported independently for PSA, imaging, and clinical measures, avoiding grouped categorizations such as complete or partial response [4]. Changes in PSA should be reported as change from baseline in a waterfall plot; bone scans are to be reported as "new lesions" or "no new lesions"; changes in soft tissue will continue to be assessed by RECIST; and pain is to be monitored using validated scales. PCWG2 also proposes that drug evaluation pathways for cytotoxic and noncytotoxic agents be developed separately. Cytotoxic drugs typically produce a decline in PSA and regression of target lesions, whereas agents that act to slow tumor growth, inhibit destruction of bone, or inhibit angiogenesis may not. Thus, PCWG2 recommends two types of phase II trial objectives: (1) those based on controlling, relieving, or eliminating disease manifestations that are present when treatment is initiated and (2) those based on preventing or delaying future disease manifestations. Following these recommendations will be critical to ensure the timely and safe development of novel therapies for CRPC.

\section{Future Directions}

While great progress has been made in the treatment of CRPC, there remains a need for better therapies that are more effective with fewer side effects. The development of new therapies for CPRC has been challenged by multiple setbacks, as trials have failed to demonstrate efficacy (e.g., atrasentan) or have been terminated prematurely because of excessive deaths (e.g., GVAX in the VITAL-2 trial). With learnings from previous study limitations, ongoing phase III trials in CRPC (Table I) have been designed with the goals of improving efficacy and minimizing AEs, as well as improving outcomes relative to existing standards of care.

A major challenge to the development of new therapeutics for CRPC is the lack of surrogate endpoints. Many current trials rely on OS as an efficacy measure, an endpoint that requires much time to assess. Additionally, as more patients are treated with combination therapies, which can include up to three different agents, it becomes more difficult to ascribe from which agent clinical benefit is derived. It is the authors' opinion that bone metastasis-free survival may be an informative surrogate endpoint to consider in efficacy analysis of treatment regimens in clinical studies.

Immune monitoring is critical in the assessment of immunotherapeutic approaches. Although clinical research to date has not demonstrated conclusive support for immune monitoring approaches, a variety of bioassaying techniques exist that may have potential for use in immune monitoring, including the enzyme-linked immunosorbent spot assay and cytometry-based tests such as intracellular cytokine staining. These techniques are starting to be employed in phase I/II trials to investigate particular immune monitoring strategies for developmental therapies. Monitoring the immune system for drug-related AEs is imperative, as the immunotherapies discussed in this review present a spectrum of toxicities. For instance, clinical trials with sipuleucel-T have demonstrated limited toxicity [30], while ipilimumab use has been reported to have more systemic side effects due to its mechanism of action [59]. Additionally, for almost all of these therapies, the patient 
Table I Ongoing phase III trials in CRPC

\begin{tabular}{|c|c|c|c|c|}
\hline $\begin{array}{l}\text { Clinical Trials. } \\
\text { gov identifier }\end{array}$ & Experimental versus control & $\begin{array}{l}\text { Primary outcome } \\
\text { measure }\end{array}$ & Patients & Sponsor \\
\hline $\begin{array}{l}\text { NCT01308580 } \\
\text { (PROSELICA) }\end{array}$ & $\begin{array}{l}20 \mathrm{mg} / \mathrm{m}^{2} \text { cabazitaxel } / \text { prednisone versus } \\
25 \mathrm{mg} / \mathrm{m}^{2} \text { cabazitaxel } / \text { prednisone }\end{array}$ & OS & $\begin{array}{l}\text { Metastatic CRPC } \\
\text { post-docetaxel }\end{array}$ & Sanofi-aventis \\
\hline $\begin{array}{r}\text { NCT01308567 } \\
\text { (FIRSTANA) }\end{array}$ & $\begin{array}{l}20 \mathrm{mg} / \mathrm{m}^{2} \text { cabazitaxel/prednisone versus } \\
25 \mathrm{mg} / \mathrm{m}^{2} \text { cabazitaxel/prednisone } \\
\text { versus docetaxel/prednisone }\end{array}$ & OS & Chemotherapy-naive metastatic CRPC & Sanofi-aventis \\
\hline NCT00887198 & $\begin{array}{l}\text { Abiraterone acetate/prednisone versus } \\
\text { placebo/prednisone }\end{array}$ & OS, PFS & $\begin{array}{l}\text { Asymptomatic or mildly symptomatic } \\
\text { chemotherapy-naive metastatic CRPC }\end{array}$ & $\begin{array}{l}\text { Cougar Biotechnology, } \\
\text { Inc. }\end{array}$ \\
\hline NCT00638690 & $\begin{array}{l}\text { Abiraterone acetate/prednisone versus } \\
\text { placebo/prednisone }\end{array}$ & OS & Metastatic CRPC post-docetaxel & $\begin{array}{l}\text { Cougar Biotechnology, } \\
\text { Inc. }\end{array}$ \\
\hline $\begin{array}{l}\text { NCT00974311 } \\
\text { (AFFIRM) }\end{array}$ & MDV3100 versus placebo & OS & Metastatic CRPC post-docetaxel & Medivation, Inc. \\
\hline $\begin{array}{l}\text { NCT01212991 } \\
\text { (PREVAIL) }\end{array}$ & MDV3100 versus placebo & OS, PFS & $\begin{array}{l}\text { Chemotherapy-naive metastatic } \\
\text { CRPC }\end{array}$ & Medivation, Inc. \\
\hline NCT01193244 & $\begin{array}{l}\text { TAK-700/prednisone versus } \\
\text { placebo/prednisone }\end{array}$ & rPFS, OS & $\begin{array}{l}\text { Chemotherapy-naive metastatic } \\
\text { CRPC }\end{array}$ & $\begin{array}{l}\text { Millennium } \\
\text { Pharmaceuticals, Inc. }\end{array}$ \\
\hline NCT01193257 & $\begin{array}{l}\text { TAK-700/prednisone versus } \\
\text { placebo/prednisone }\end{array}$ & OS & Metastatic CRPC post-docetaxel & $\begin{array}{l}\text { Millennium } \\
\text { Pharmaceuticals, Inc. }\end{array}$ \\
\hline NCT01057810 & Ipilimumab versus placebo & OS & $\begin{array}{l}\text { Asymptomatic or minimally } \\
\text { symptomatic chemotherapy-naive } \\
\text { metastatic CRPC }\end{array}$ & Bristol-Myers Squibb \\
\hline NCT00861614 & $\begin{array}{l}\text { Ipilimumab versus placebo, each } \\
\text { following a single dose of RT }\end{array}$ & OS & Metastatic CRPC post-docetaxel & Bristol-Myers Squibb \\
\hline
\end{tabular}

$C R P C$ castration-resistant prostate cancer, $O S$ overall survival, $P F S$ progression-free survival, $r P F S$ radiographic progression-free survival, $R T$ radiotherapy

population best suited for intervention has not been clearly defined.

Another challenge is finding the right combination and timing of therapy for CRPC because of the complexity in testing each combination and other intellectual property issues. The dose range for chemotherapy, when combined with immunotherapy, has proven to be surprisingly low and the effects are critically dependent on the relative timing of the two agents. Furthermore, to date, most novel agents such as immunotherapy have been introduced late in the disease, at a time when the tumor has already developed multiple mechanisms to evade the immune system. Recent work has identified a few biochemical markers (PSA doubling time, cancer-testis antigen and neutral endopeptidase expression, and AR polymorphisms) [60-63] that show increased expression in patients that are likely to develop CRPC. Defining key biomarkers will help identify patient populations that may benefit from earlier treatment intervention, as well as patients that may respond to specific molecular agents.

An additional reason that therapies have failed to meet efficacy endpoints is because the biology behind the development and progression of CRPC is not well defined. Furthermore, preclinical models used to model CRPC are not representative of the clinical course of the disease. More research in CRPC, including the development of new cell lines and prostate tumor models, are needed to test novel therapies.
Lastly, clinical trial design needs to be improved to maximize its impact. Trials must be standardized to compare treatment regimens; for example, if chemotherapy is to be the comparator arm (instead of placebo), it needs to be standardized across treatment groups. In addition, the metrics currently used for measuring response (e.g., PSA decline) may not correlate with a clinically meaningful outcome; OS is likely a better primary endpoint to evaluate clinical activity. The direction of therapy design should be carefully assessed and reevaluated as our understanding of the pathology of CRPC and the technology for developing new therapies evolves. Unlike chemotherapy, immunotherapy requires time to take effect as a patient's immune system must mount a response to the tumor. Thus, trials of immunotherapeutic agents need to allow for the immune system response before measuring the antitumor response. In addition, the use of immunotherapies may require a somewhat revised set of guidelines with which to evaluate responses, as this group of agents often demonstrates response patterns not captured by standard RECIST or World Health Organization criteria [64].

The addition of immunotherapy to the therapeutic options for CRPC is likely to greatly improve efficacy and safety for this difficult-to-treat disease. Combinations of immunomodulators, vaccines, antibodies, and chemotherapy will most likely be needed for the host to mount a more vigorous antitumor response for patients with ad- 
vanced disease. By employing combinations of agents, such treatment regimens may impact the intratumor balance to shift in favor of immune response. For example, certain combinations of immunotherapy with chemotherapy agents or vaccines may be pivotal in the elimination of immunosuppressive regulatory $\mathrm{T}$ cells, in turn increasing the ratio in support of effector $\mathrm{T}$ cells to mediate antitumor immunity.

Currently, much work has been focused on enhancing vaccine therapy by adding another immunotherapy such as a checkpoint blockade agent or some conventional therapy like radiation or chemotherapy. However, single-agent checkpoint blockade has shown dramatic RECIST responses in melanoma [64] and more recently in prostate cancer. These recent findings are a reason to rethink that dogma and instead investigate how to enhance a checkpoint blockade therapy (e.g., ipilimumab) by the addition of another checkpoint blocker, chemotherapy, or radiation. The timing of immunotherapy will likely prove crucial; patients will likely respond better if treated at an earlier time point, before the tumor develops multiple ways to evade the immune system. Finding the right combinations and sequences that maximize efficacy while minimizing toxicity will be challenging and take extensive and careful research.

Acknowledgments Dr. Sharma was supported in part by a Challenge Grant in Immunology from the Prostate Cancer Foundation and a Mentored Scholar Grant from the American Cancer Society. The authors wish to thank Charles Drake, MD, $\mathrm{PhD}$, of the Johns Hopkins Sidney Kimmel Comprehensive Cancer Center for his contributions to scientific discussion and critical review of the manuscript. Writing and editorial support was provided by StemScientific, funded by BristolMyers Squibb Company.

Conflict of Interest WRG participates in several advisory boards for Bayer, Novartis, Morphotec, Bristol-Meyers Squibb, Sanofiaventis, Aglaia Biomedical Ventures. PS participated on several advisory boards for Bristol-Meyers Squibb.

Open Access This article is distributed under the terms of the Creative Commons Attribution Noncommercial License which permits any noncommercial use, distribution, and reproduction in any medium, provided the original author(s) and source are credited.

\section{References}

1. Jemal A, Siegel R, Ward E, Hao Y, Xu J, Thun MJ. Cancer statistics. CA Cancer J Clin. 2009;59:225-49.

2. American Urological Association. The management of localized prostate cancer: patient guide. http://www.auanet.org/content/ guidelines-and-quality-care/clinical-guidelines/patient-guides/ pc08.pdf. Accessed 14 Mar 2010.

3. Risk M, Corman JM. The role of immunotherapy in prostate cancer: an overview of current approaches in development. Rev Urol. 2009;11:16-27.

4. Scher HI, Halabi S, Tannock I, Morris M, Sternberg CN, Carducci MA, et al. Prostate Cancer Clinical Trials Working Group. Design and end points of clinical trials for patients with progressive prostate cancer and castrate levels of testosterone: recommendations of the Prostate Cancer Clinical Trials Working Group. J Clin Oncol. 2008;26:1148-59.

5. Tannock IF, de Wit R, Berry WR, Horti J, Pluzanska A, Chi KN, et al. TAX 327 Investigators. Docetaxel plus prednisone or mitoxantrone plus prednisone for advanced prostate cancer. $\mathrm{N}$ Engl J Med. 2004;351:1502-12.

6. Petrylak DP, Tangen CM, Hussain MH, Lara Jr PN, Jones JA, Taplin ME, et al. Docetaxel and estramustine compared with mitoxantrone and prednisone for advanced refractory prostate cancer. N Engl J Med. 2004;351:1513-20.

7. Tannock IF, Osoba D, Stockler MR, Ernst DS, Neville AJ, Moore $\mathrm{MJ}$, et al. Chemotherapy with mitoxantrone plus prednisone or prednisone alone for symptomatic hormone-resistant prostate cancer: a Canadian randomized trial with palliative end points. J Clin Oncol. 1996;14:1756-64.

8. de Bono JS, Oudard S, Ozguroglu M, Hansen S, Machiels JP, Kocak I, et al. For the TROPIC Investigators. Prednisone plus cabazitaxel or mitoxantrone for metastatic castration-resistant prostate cancer progressing after docetaxel treatment: a randomised open-label trial. Lancet. 2010;376:1147-54.

9. de Bono JS, Logothetis CJ, Fizazi K, North S, Chu L, Chi K, et al. Abiraterone acetate (AA) plus low dose prednisone (P) improves overall survival (OS) in patients with metastatic castrationresistant prostate cancer (MCRPC) who have progressed after docetaxel-based chemotherapy: results of COU-AA-301, a randomized double-blind placebo-controlled phase 3 study. Ann Oncol. 2010;21 Suppl 8:viiii3. Abstract LBA5.

10. Tran C, Ouk S, Clegg NJ, Chen Y, Watson PA, Arora V, et al. Development of a second-generation antiandrogen for treatment of advanced prostate cancer. Science. 2009;324:787-90.

11. Hirmand M, Scher HI, Beer T, Higano CS, Taplin M-E, Efstathiou E. Circulating tumor cells (CTC) and prostate-specific antigen (PSA) as response indicator biomarkers in chemotherapy-naïve patients with progressive castration-resistant prostate cancer (CRPC) treated with MDV3100. Ann Oncol. 2010;21:viii277.

12. Beltran H, Beer TM, Carducci MA, de Bono J, Gleave M, Hussain $M$, et al. New therapies for castration-resistant prostate cancer: efficacy and safety. Eur Urol. 2011;60:279-90.

13. Hussain M, Tangen CM, Lara Jr PN, Vaishampayan UN, Petrylak $\mathrm{DP}$, Colevas $\mathrm{AD}$, et al. Ixabepilone (epothilone B analogue BMS247550 ) is active in chemotherapy-naive patients with hormonerefractory prostate cancer: a Southwest Oncology Group trial S0111. J Clin Oncol. 2005;23:8724-9.

14. Coffey DS, Isaacs JT. Prostate tumor biology and cell kineticstheory. Urology. 1981;17:40-53.

15. Mercader M, Bodner BK, Moser MT, Kwon PS, Park ES, Manecke RG, et al. T cell infiltration of the prostate induced by androgen withdrawal in patients with prostate cancer. Proc Natl Acad Sci U S A. 2001;98:14565-70.

16. Sfanos KS, Bruno TC, Meeker AK, De Marzo AM, Isaacs WB, Drake CG. Human prostate-infiltrating CD8+ T lymphocytes are oligoclonal and PD-1+. Prostate. 2009;69:1694-703.

17. Bander NH, Milowsky MI, Nanus DM, Kostakoglu L, Vallabhajosula S, Goldsmith SJ. Phase I trial of 177lutetium-labeled J591, a monoclonal antibody to prostate-specific membrane antigen, in patients with androgen independent prostate cancer. J Clin Oncol. 2005;23:4591-601.

18. Milowsky MI, Nanus DM, Kostakoglu L, Vallabhajosula S, Goldsmith SJ, Bander NH. Phase I trial of yttrium-90-labeled anti-prostate specific membrane antigen monoclonal antibody $\mathrm{J} 591$ for androgen-independent prostate cancer. J Clin Oncol. 2004;22:2522-31.

19. Tagawa ST, Milowsky MI, Morris MJ, Vallabhajosula S, Goldsmith S, Matulich D, et al. Phase II trial of 177lutetium 
radiolabeled anti-prostate-specific membrane antigen (PSMA) monoclonal antibody J591 (177Lu-J591) in patients (pts) with metastatic castrate-resistant prostate cancer (metCRPC). J Clin Oncol. 2008;26(Suppl):15s. Abstract 5140.

20. Sanda MG, Smith DC, Charles LG, Hwang C, Pienta KJ, Schlom $\mathrm{J}$, et al. Recombinant vaccinia-PSA (PROSTVAC) can induce a prostate-specific immune response in androgen-modulated human prostate cancer. Urology. 1999;53:260-6.

21. Eder JP, Kantoff PW, Roper K, Xu GX, Bubley GJ, Boyden J, et al. A phase I trial of a recombinant vaccinia virus expressing prostate-specific antigen in advanced prostate cancer. Clin Cancer Res. 2000;6:1632-8.

22. Kaufman HL, Wang W, Manola J, DiPaola RS, Ko YJ, Sweeney $\mathrm{C}$, et al. Phase II randomized study of vaccine treatment of advanced prostate cancer (E7897): a trial of the Eastern Cooperative Oncology Group. J Clin Oncol. 2004;22:2122-32.

23. DiPaola RS, Plante M, Kaufman H, Petrylak DP, Israeli R, Lattime E, et al. A phase I trial of pox PSA vaccines (PROSTVAC-VF) with B7-1, ICAM-1, and LFA-3 costimulatory molecules (TRICOM) in patients with prostate cancer. J Transl Med. 2006;4:1.

24. May Jr KF, Gulley JL, Drake CG, Dranoff G, Kantoff PW. Prostate cancer immunotherapy. Clin Cancer Res. 2011;17 (16):5233-8.

25. Kantoff PW, Schuetz TJ, Blumenstein BA, Glode LM, Bilhartz DL, Wyand $\mathrm{M}$, et al. Overall survival analysis of a phase II randomized controlled trial of a poxviral-based PSA-targeted immunotherapy in metastatic castration-resistant prostate cancer. J Clin Oncol. 2010;28:1099-105.

26. Higano CS, Corman JM, Smith DC, Centeno AS, Steidle CP, Gittleman M, et al. Phase 1/2 dose-escalation study of a GM-CSFsecreting, allogeneic, cellular immunotherapy for metastatic hormone-refractory prostate cancer. Cancer. 2008;113:975-84.

27. Small E, Demkow T, Gerritsen WR, Rolland F, Hoskin P, Smith DC, et al. A phase III trial of GVAX immunotherapy for prostate cancer in combination with docetaxel versus docetaxel plus prednisone in symptomatic, castration-resistant prostate cancer (CRPC). Presented at the American Society of Clinical Oncology 2009 Genitourinary Cancers Symposium; February 26-28, 2009; Orlando, FL, USA. Abstract 7.

28. McNeel DG, Dunphy EJ, Davies JG, Frye TP, Johnson LE, Staab MJ, et al. Safety and immunological efficacy of a DNA vaccine encoding prostatic acid phosphatase in patients with stage D0 prostate cancer. J Clin Oncol. 2009;27:4047-54.

29. Small EJ, Schellhammer PF, Higano CS, Redfern CH, Nemunaitis JJ, Valone FH, et al. Results of a placebo-controlled phase III trial of immunotherapy with APC8015 for patients with hormone refractory prostate cancer (HRPC). J Clin Oncol. 2005;23:16s. Abstract 4500.

30. Kantoff P, Higano CS, Shore ND, Berger ER, Small EJ, Penson $\mathrm{DF}$, et al. Sipuleucel-T immunotherapy for metastatic castrationresistant prostate cancer. N Engl J Med. 2010;363:411-22.

31. Small EJ, Reese DM, Um B, Whisenant S, Dixon SC, Figg WD. Therapy of advanced prostate cancer with granulocyte macrophage colony-stimulating factor. Clin Cancer Res. 1999;5:173844.

32. Rini BI, Weinberg V, Bok R, Small EJ. Prostate-specific antigen kinetics as a measure of the biologic effect of granulocytemacrophage colony-stimulating factor in patients with serologic progression of prostate cancer. J Clin Oncol. 2003;21:99-105.

33. Chambers CA, Krummel MF, Boitel B, Hurwitz A, Sullivan TJ, Fournier S, et al. The role of CTLA- 4 in the regulation and initiation of T-cell responses. Immunol Rev. 1996;153:2746.

34. Liakou CI, Kamat A, Tang DN, Chen H, Sun J, Troncoso P, et al. CTLA-4 blockade increases IFNgamma-producing $\mathrm{CD}^{+}{ }^{+} \mathrm{ICOS}^{\text {hi }}$ cells to shift the ratio of effector to regulatory $\mathrm{T}$ cells in cancer patients. Proc Natl Acad Sci U S A. 2008;105:14987-92.

35. Chen H, Liakou CI, Kamat A, Pettaway C, Ward JF, Tang DN, et al. Anti-CTLA-4 therapy results in higher $\mathrm{CD}^{+} \mathrm{ICOS}^{\text {hi }} \mathrm{T}$ cell frequency and IFN- $\gamma$ levels in both nonmalignant and malignant prostate tissues. Proc Natl Acad Sci U S A. 2009;106:2729-34.

36. Small EJ, Tchekmedyian NS, Rini BI, Fong L, Lowy I, Allison JP. A pilot trial of CTLA-4 blockade with human anti-CTLA-4 in patients with hormone-refractory prostate cancer. Clin Cancer Res. 2007;13:1810-5.

37. Fong L, Kwek SS, O'Brien S, Kavanagh B, McNeel DG, Weinberg V, et al. Potentiating endogenous antitumor immunity to prostate cancer through combination immunotherapy with CTLA4 blockade and GM-CSF. Cancer Res. 2009;69:609-15.

38. Beer TM, Slovin SF, Higano CS, Tejwani S, Dorff TB, Stankevich E, et al. Prostate Cancer Clinical Trials Consortium. Phase I trial of ipilimumab (IPI) alone and in combination with radiotherapy (XRT) in patients with metastatic castration resistant prostate cancer (mCRPC). J Clin Oncol. 2008;26(Suppl):15s Abstract 5004.

39. Slovin SF, Beer TM, Higano CS, Tejwani S, Hamid O, Picus J, et al. Prostate Cancer Clinical Trials Consortium. Initial phase II experience of ipilimumab (IPI) alone and in combination with radiotherapy (XRT) in patients with metastatic castration-resistant prostate cancer (mCRPC). J Clin Oncol. 2009;27(Suppl):15s Abstract 5138.

40. Brahmer JR, Drake CG, Wollner I, Powderly JD, Picus J, Sharfman WH, et al. Phase I study of single-agent antiprogrammed death-1 (MDX-1106) in refractory solid tumors: safety, clinical activity, pharmacodynamics, and immunologic correlates. J Clin Oncol. 2010;28:3167-75.

41. Sznol M, Powderly JD, Smith DC, Brahmer JR, Drake CG, McDermott DF, et al. Safety and antitumor activity of biweekly MDX-1106 (Anti-PD-1, BMS-936558/ONO-4538) in patients with advanced refractory malignancies. J Clin Oncol. 2010;28 (Suppl):15s. Abstract 2506.

42. Arlen PM, Gulley JL, Parker C, Skarupa L, Pazdur M, Panicali D, et al. A randomized phase II study of concurrent docetaxel plus vaccine versus vaccine alone in metastatic androgen-independent prostate cancer. Clin Cancer Res. 2006;12:1260-9.

43. Petrylak DP, Small E, Schellhammer PF. Androgen independent prostate cancer (AIPC) patients who receive sipuleucel-T followed by docetaxel have prolonged survival. Presented at the American Urological Association 2007 Annual Meeting; May 19-24, 2007; Anaheim, CA, USA. Abstract 605.

44. Morse MD, McNeel DG. Prostate cancer patients on androgen deprivation therapy develop persistent changes in adaptive immune responses. Hum Immunol. 2010;71:496-504.

45. Tollefson MK, Karnes RJ, Thompson RH, Granberg CF, Hillman DW, Breau RH, et al. A randomized phase II study of ipilimumab with androgen ablation compared with androgen ablation alone in patients with advanced prostate cancer. Presented at the American Society of Clinical Oncology 2010 Genitourinary Cancers Symposium; March 5-7, 2010; San Francisco, CA, USA. Abstract 168.

46. Madan RA, Gulley JL, Schlom J, Steinberg SM, Liewehr DJ, Dahut WL, et al. Analysis of overall survival in patients with nonmetastatic castration-resistant prostate cancer treated with vaccine, nilutamide, and combination therapy. Clin Cancer Res. 2008;14:4526-31.

47. Nesslinger NJ, Sahota RA, Stone B, Johnson K, Chima N, King $C$, et al. Standard treatments induce antigen-specific immune responses in prostate cancer. Clin Cancer Res. 2007;13:1493-502.

48. Apetoh L, Ghiringhelli F, Tesniere A, Obeid M, Ortiz C, Criollo A, et al. Toll-like receptor 4-dependent contribution of the 
immune system to anticancer chemotherapy and radiotherapy. Nature Med. 2007;13:1050-9.

49. Lugade AA, Moran JP, Gerber SA, Rose RC, Frelinger JG, Lord EM. Local radiation therapy of B16 melanoma tumors increases the generation of tumor antigen-specific effector cells that traffic to the tumor. J Immunol. 2005;174:7516-23.

50. Gulley JL, Arlen PM, Bastian A, Morin S, Marte J, Beetham P, et al. Combining a recombinant cancer vaccine with standard definitive radiotherapy in patients with localized prostate cancer. Clin Cancer Res. 2005;11:3353-62.

51. Gerritsen W, van den Eertwegh AJ, de Gruijl T, van den Berg HP, Scheper RJ, Sacks N, et al. Expanded phase I combination trial of GVAX immunotherapy for prostate cancer and ipilimumab in patients with metastatic hormone-refractory prostate cancer (mHPRC). J Clin Oncol. 2008;26(Suppl):15s. Abstract 5146.

52. Small E, Higano C, Tchekmedyian N, Sartor O, Stein B, Young R, et al. Randomized phase II study comparing 4 monthly doses of ipilimumab (MDX-010) as a single agent or in combination with a single dose of docetaxel in patients with hormone-refractory prostate cancer. J Clin Oncol. 2006;24(Suppl):18s. Abstract 4609.

53. Hersh EM, O'Day SJ, Powderly J, Khan KD, Pavlick AC, Cranmer LD, et al. A phase II multicenter study of ipilimumab with or without dacarbazine in chemotherapy-naïve patients with advanced melanoma. Invest New Drugs. 2011;29:489-98.

54. Sternberg CN, Whelan P, Hetherington J, Paluchowska B, Slee PH, Vekemans K, et al. Genitourinary Tract Group of the EORTC. Phase III trial of satraplatin, an oral platinum plus prednisone vs. prednisone alone in patients with hormone-refractory prostate cancer. Oncology. 2005;68:2-9.

55. Sartor AO, Petrylak DP, Witjes JA, Berry WR, Chatta GS, Vaughn DJ, et al. Satraplatin in patients with advanced hormone-refractory prostate cancer (HRPC): overall survival (OS) results from the phase III satraplatin and prednisone against refractory cancer (SPARC) trial. J Clin Oncol. 2008;26(Suppl):15s. Abstract 5003.

56. Nelson JB, Love W, Chin JL, Saad F, Schulman CC, Sleep DJ, et al. Atrasentan Phase 3 Study Group. Phase 3, randomized, controlled trial of atrasentan in patients with nonmetastatic, hormone-refractory prostate cancer. Cancer. 2008;113:2478-87.

57. Armstrong AJ, Creel P, Turnbull J, Moore C, Jaffe TA, Haley S, et al. A phase I-II study of docetaxel and atrasentan in men with castration-resistant metastatic prostate cancer. Clin Cancer Res. 2008;14:6270-6.

58. Scher HI, Chi KN, De Wit R, Berry WR, Albers P, Henick B, et al. Docetaxel (D) plus high-dose calcitriol versus D plus prednisone $(\mathrm{P})$ for patients $(\mathrm{Pts})$ with progressive castrationresistant prostate cancer (CRPC): results from the phase III ASCENT2 trial. J Clin Oncol. 2010;28(Suppl):15s. Abstract 4509.

59. Hodi FS, O’Day SJ, McDermott DF, Weber RW, Sosman JA, Haanen JB, et al. Improved survival with ipilimumab in patients with metastatic melanoma. N Engl J Med. 2010;363:711-23.

60. Albiges L, Prapotnich D, Fizazi D, Cathelineau X, Massard C, Mombet A, et al. PSA doubling time is the strongest predictor for metastases, castration resistance, and death in patients with prostate cancer treated with intermittent androgen deprivation for isolated biochemical relapse. Ann Oncol. 2010;21:vii272.

61. von Boehmer L, Keller L, Mortezavi A, Provenzano M, Sais G, Hermanns $\mathrm{T}$, et al. MAGE-C2/CT10 protein expression is an independent predictor of recurrence in prostate cancer. Ann Oncol. 2010;21:vii273.

62. Vlachostergios PJ, Patrikidou A, Kakkas G, Moutzouris G, Voutsadakis IA, Karasavvidou F, et al. Neutral endopeptidase (NEP) and endothelin 1 (ET-1) are inversely expressed in hormone-naïve prostate cancer and are predictors of biochemical failure from radical prostatectomy. Ann Oncol. 2010;21:vii278.

63. Sousa M, Ribeiro R, Mauricio MJ, Begonha R, Monteiro C, Cunha V, et al. Pharmacogenomic profile of castration-resistant prostate cancer: the influence of $\mathrm{AR}+1733 \mathrm{G}>\mathrm{A}$ and $\mathrm{SHBG}$ $+5790 \mathrm{G}>$ A polymorphisms. Ann Oncol. 2010;21:vii280.

64. Wolchok J, Hoos A, O’Day S, Weber JS, Hamid O, Lebbé C, et al. Guidelines for the evaluation of immune therapy activity in solid tumors: immune-related response criteria. Clin Cancer Res. 2009;15:7412-20. 